\title{
Rigid and elastic registration for coronary artery IVUS images
}

\author{
Zheng Sun*, Hua Bai and Bingru Liu \\ Department of Electronic and Communication Engineering, North China Electric Power University, \\ Baoding, Hebei, China
}

\begin{abstract}
.
BACKGROUND: Intravascular ultrasound (IVUS) has been widely used in diagnosis and interventional treatment of cardiac vessel diseases. The coronary artery IVUS images are usually polluted by motion artifacts caused by cardiac motion, pulsatile blood and catheter twist during continuous pullback acquisition.

OBJECTIVE: Strategies for rigid and elastic registration of coronary artery IVUS studies are developed to suppress the longitudinal motion and misalignment between successive frames.

METHODS: Rigid registration is performed by searching for the optimal matching for each frame in other cycles based on the cyclic variation of gray-scale features. The image sequence is gated to properly identify the frames in each cardiac phase. Then, elastic registration between frames is achieved through an optimization algorithm based on thin plate spline (TPS) to correct the misalignment of successive slices.

RESULTS: Experimental results with in vivo image data shows that the rigid registration performs better than the offline ECG gating. The elastic mapping relation between lumen contours in successive frames is smooth and continuous.

CONCLUSION: The serrated vessel wall borders in longitudinal cuts are smoothed after rigid registration while image segmentation and feature extraction are required. The point-to-point correspondence between lumen contours detected from two matched frames is obtained with elastic registration.
\end{abstract}

Keywords: Coronary artery, intravascular ultrasound (IVUS), motion artifact, image misalignment, rigid registration, elastic registration

\section{Introduction}

Intravascular ultrasound (IVUS) is a catheter based imaging modality that has been widely applied in clinical diagnosis and interventional treatment of coronary artery atherosclerosis. An ultrasonic transducer mounted on the tip of a special catheter is directly inserted into the vascular lumen and pushed to the distal end. Then, during slow pullback of the catheter at a constant speed, a series of tomographic gray-scale images recording the luminal cross-sections are acquired. The cross-sectional images display three ring-like layers of the vessel wall around the imaging catheter, i.e., intima, media, adventitia and adjacent structures. Sixty successive frames are acquired for a vessel segment of about $1 \mathrm{~mm}$ at the pullback speed of $0.5 \mathrm{~mm} / \mathrm{s}$ and the frame rate of $30 \mathrm{fps}$. In consequence, a strong correlation is seen between consecutive frames and their appearances are very similar.

During the continuous pullback acquisition of coronary artery IVUS studies, the spatial consistency between consecutive frames is corrupted by the vessel wall displacement, periodical change in vascular

\footnotetext{
${ }^{*}$ Corresponding author: Sun Zheng, Department of Electronic and Communication Engineering, North China Electric Power University, Baoding 071003, Hebei, China. E-mail: sunzheng_tju@163.com.
} 


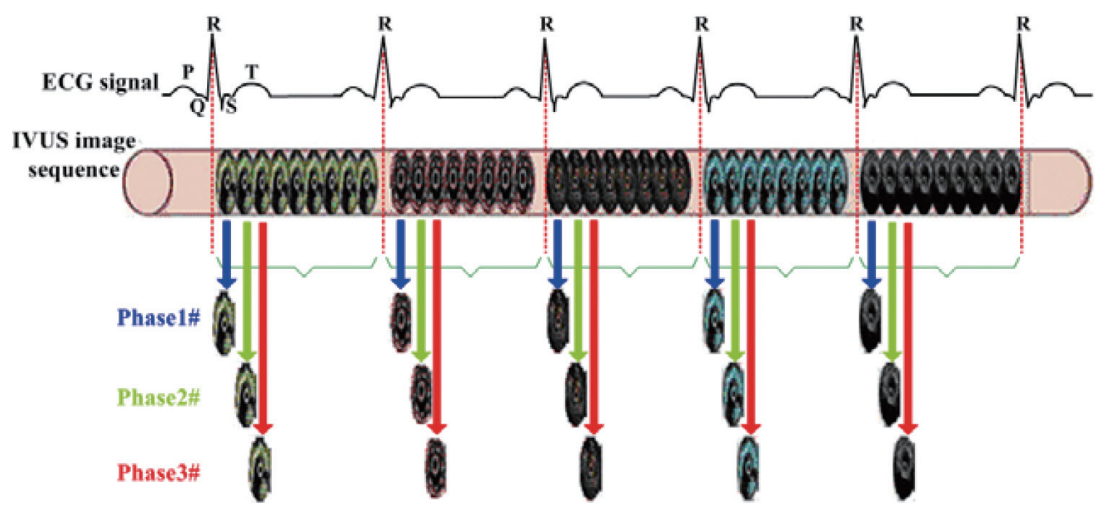

Fig. 1. Schematic diagram of rigid registration of an IVUS study.

dimensions and the catheter movement relative to the vessel wall caused by pulsatile blood and cardiac motion. It is known as motion artifact, which is decomposed into two spatial components, longitudinal (or axial) motion and in-plane motion [1,2]. The former relates to the forward or backward longitudinal displacement of the catheter along the vessel axis. It leads to a "saw-tooth-shaped" appearance of the vessel wall in IVUS longitudinal cuts. The latter relates to the relative translation and rotation between the ultrasonic transducer and the lumen. It produces the misalignment of the vessel structures from one cross-sectional image to the other. Quantitative geometrical characterization and vessel reconstruction are hindered for non-diastolic phases since the vascular cross-sections are not equally located in space along the catheter pullback trajectory. The longitudinal motion and misalignment of frames should be corrected to improve the visualization of the longitudinal cuts and ensure the accuracy of quantitative measures and 3-D reconstruction.

One of widely used strategies to reduce longitudinal motion artifacts is to synchronize IVUS image acquisition by an online ECG-gating device [3]. However, most clinically used IVUS imaging systems are not equipped with a special gating acquisition device. Furthermore, the cardiac intervention may be significantly prolonged due to one frame per cycle. The retrospective offline ECG gating is subjective and sensitive to intra-operator variation as well as inter-operator or patient variation [4]. Furthermore, only approximate synchronization can be obtained since the frame rate is fixed,

Past techniques for suppressing in-plane motion artifacts estimate the displacements between the geometrical centers of the luminal cross-sections and determine the rotation angles by Fourier spectrum analyses $[2,5,6]$. However, one-to-one point matching between the lumen contours in adjacent frames can not be achieved.

In the present work, the problem of rigid and elastic registration for a continuous pullback IVUS study is addressed. Rigid registration is performed by searching for the optimal matching for each frame in other heart periods based on the cyclic variation of gray-scale features. For each gated sub-sequence, the elastic registration is performed by finding the spatial mapping relation between the detected lumen contours with an optimization algorithm based on thin plate spline (TPS) interpolation. The misalignment of successive cross-sectional frames is thus corrected and their spatial consistency is achieved. Both in-plane and longitudinal motion components are suitably suppressed.

The rest of this paper is organized as follows: the details of the proposed methods are introduced in Sections 2 and 3; Section 4 gives experimental results validating the methods. Conclusion and summary are described in Section 5. 


\section{Rigid registration}

The image features of coronary artery IVUS studies present cyclic changes. Therefore, they imply information of cardiac phases that can be used to gate the image sequence. First, the cyclic changes of image intensities along the overall cross-sectional image sequence are described with a dissimilarity matrix. Then, a path with the smallest accumulative dissimilarity is found within the dissimilarity matrix to determine the matching for a frame in the subsequent periods. In consequence, the image sequence is gated into several sub-sequences and each corresponds to a specific cardiac phase (see Fig. 1). Thus, the serrated vessel wall borders in the longitudinal cuts are smoothed.

\subsection{Constructing dissimilarity matrix}

A $n \times n$ matrix, $\boldsymbol{D}$, is constructed for an IVUS study, $\left\{I_{1}, I_{2}, \ldots, I_{n}\right\}$. Each element of $\boldsymbol{D}, d_{i, j}$, is the difference between $I_{i}$ and $I_{j}$ based on the normalized cross correlation of the intensity features [7],

$$
d_{i, j}=1-\frac{\sum_{m=0}^{N_{1}-1} \sum_{n=0}^{N_{2}-1}\left|I_{i}(m, n)-\bar{I}_{i}\right| \cdot\left|I_{j}(m, n)-\bar{I}_{j}\right|}{\sqrt{\left[\sum_{m=0}^{N_{1}-1} \sum_{n=0}^{N_{2}-1}\left[I_{i}(m, n)-\bar{I}_{i}\right]^{2}\right]\left[\sum_{m=0}^{N_{1}-1} \sum_{n=0}^{N_{2}-1}\left[I_{j}(m, n)-\bar{I}_{j}\right]^{2}\right]}},
$$

where, $i, j=1,2, \ldots, N$, and $\bar{I}_{i}$ and $\bar{I}_{j}$ are the average intensity of $I_{i}$ and $I_{j}$. A small $d_{i, j}$ indicates that the image contents of $I_{i}$ and $I_{j}$ are similar.

\subsection{Determining the starting point and searching scope of the optimal path}

The appearances of matching frames acquired in the same cardiac phase of adjacent cycles are very similar. Therefore, the dissimilarity between them should present a local minimum in $\boldsymbol{D}$. The correspondent frames of successive cycles are detected through finding a path with the minimal accumulated dissimilarity within $\boldsymbol{D}$.

The first row of $\boldsymbol{D},\left[d_{1,1}, d_{1,2}, \ldots, d_{1, n}\right]$, where $d_{1,1}=0$, are the differences between the first frame, $I_{1}$, and other frames. The profile $d_{1, i}(i=1,2, \ldots, n)$ presents nearly cyclic structure depending on cardiac cycles. Its successive local extrema are nearly equal because changes in the appearances of IVUS frames mainly depend on cardiac cycles. Also, local extrema tend to increase progressively with $i$. $i$ of the first local extremum except $d_{1,1}=0$ is the frame number of the matching of $I_{1}$ in the adjacent cycle, which is represented as $F$. Then, the optimal path origins from $d_{1, F}$.

In clinic, the imaging catheter is always drawn back at a constant speed acquiring tomographic images at a constant frame rate. If the heart rate is nearly invariable during the intervention, the cardiac cycle length in frames, $C_{0}$, should be equal to the frame number difference between matched frames in consecutive cardiac periods. However, the heart rate may fluctuate during the cardiac catheterization. Therefore, the searching range of the optimal path, $\Delta$, is set as $C_{0} / 2 \leqslant \Delta \leqslant 2 C_{0}[6,8]$. That is, if $I_{r}$ matches with $I_{k}$ in the next cycle, $k$ should range in $[r, r+\Delta]$.

\subsection{Finding an optimal path}

As shown in Fig. 2, a node in $\boldsymbol{D}, d_{i, j}$, relates to $I_{i}$ and $I_{j} . L_{2}$ is the main diagonal and the dissimilarity values of those nodes on it are 0 . An optimal path, $L$, originating from $d_{1, F}$, with the lowest accumulative dissimilarity is searched within the region between $L_{1}$ and $L_{2}$ with dynamic programming algorithm [9] to achieve a global optimum. 


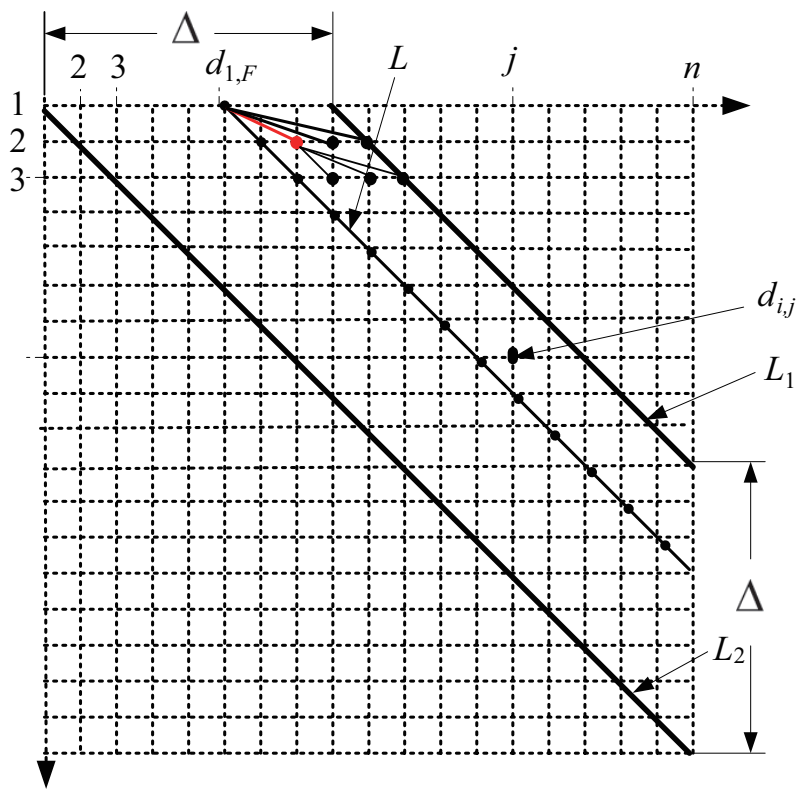

Fig. 2. A $n \times n$ dissimilarity matrix.

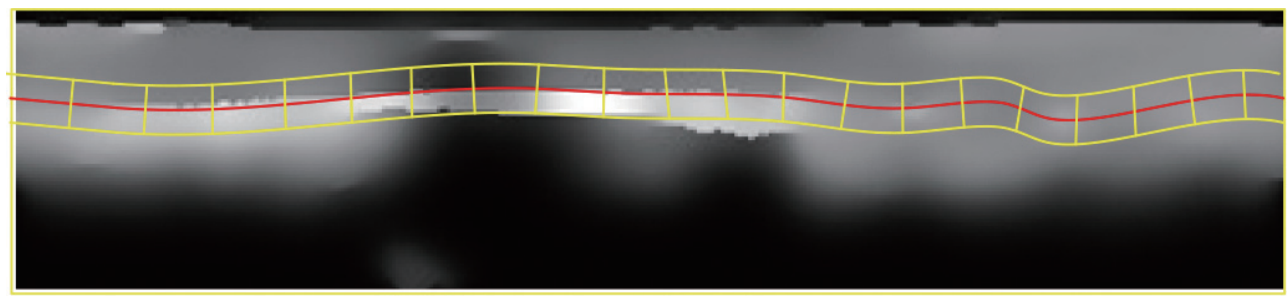

Fig. 3. Polar view of an IVUS image with the detected lumen contour.

\section{Elastic registration}

After rigid registration, the original image sequence is categorized into several sub-sequences in different cardiac phases. Although the tomographic frames within each sub-sequence are longitudinally ordered, the misalignments between consecutive frames may not be homogeneous due to the large variability in the ultrasonic transducer motion with respective to the vessel.

For each frame in a sub-sequence, the lumen contour is firstly detected [10] and the cross-sectional view is then transformed into the polar view as shown in Fig. 3. Elastic registration is performed between lumen contours in consecutive frames adopting thin plate spline (TPS) interpolation algorithm [11]. The correspondence between two sets of lumen contour points is found by minimizing TPS deformation energy in an iterative manner under the constraint of one-to-one mapping. The detailed steps are as follows.

The TPS deformation energy between lumen contour point sets, $\boldsymbol{C}_{1}=\left\{\boldsymbol{c}_{1 k} \in \boldsymbol{C}_{1}, k=1,2, \ldots, K\right\}$ and $\boldsymbol{C}_{2}=\left\{\boldsymbol{c}_{2 i} \in \boldsymbol{C}_{2}, i=1,2, \ldots, N\right\}$, is defined as

$$
E(M, f)=\sum_{i=1}^{N} \sum_{k=1}^{K} m_{k i}\left\|c_{2 i}-f\left(c_{1 k}\right)\right\|^{2}+T \sum_{i=1}^{N} \sum_{k=1}^{K} m_{k i} \lg m_{k i}-\eta \sum_{i=1}^{N} \sum_{k=1}^{K} m_{k i} .
$$


$f(\cdot)$ is the coordinate transformation function of lumen contour points,

$$
f(x, y)=a_{1}+a_{x} x+a_{y} y+\sum_{i=1}^{N} w_{i} U\left(\left|P_{i}-(x-y)\right|\right) .
$$

where, $a_{1}, a_{x}, a_{y}$ and $w_{i}(i=1,2, \ldots, N)$ are undetermined coefficients, $P_{i}=\left(x_{i}, y_{i}\right)(i=1,2, \ldots$, $N$ ) is a point belonging to the lumen contour point set to be registered, and $U(r)$ is the base function of TPS,

$$
U(r)=r^{2} \lg r^{2},
$$

where $r=\sqrt{x^{2}+y^{2}}$.

$T$ in Eq. (2) is the temperature coefficient. A large $T$ means that the constraint between a pair of lumen contour points is relatively relaxed. With the decrease of $T$, the TPS deformation energy tends to decrease gradually.

$m_{k i}$ in Eq. (2) is a mapping coefficient (i.e., match measure) between $c_{1 k}$ and $c_{2 i}$ [12],

$$
m_{k i}=\frac{1}{T} e^{-\frac{\left(c_{2 i}-f\left(c_{1 k}\right)\right)^{T}\left(c_{2 i}-f\left(c_{1 k}\right)\right)}{2 T}} .
$$

Ideally, if $c_{1 k}$ and $c_{2 i}$ are matched, $m_{k i}=1$; otherwise, $m_{k i}=0$. However, the match measure between lumen contours may not exactly be 0 or 1 . Therefore, a matching measure matrix between $C_{1}$ and $C_{2}$ is defined as $\boldsymbol{M}=\left\{\boldsymbol{m}_{k i} \in[0,1] \mid k=1,2, \ldots, K ; i=1,2, \ldots, N\right\}$ to obtain robust matching.

$\eta$ in Eq. (2) is a weight controlling the number of singularities in the lumen contour point sets to be registered. The widely accepted method to calculate such a coefficient has not existed. In this study, $T$ of those singularities is set as a very large value so that the singularity points are combined into a set.

The TPS deformation energy is minimized in an iterative manner so that the matching measure matrix and mapping coefficients between lumen contours detected from consecutive IVUS frames are jointly solved. $T$ should be initiated at the beginning of the iteration. Then, during the iterative process, $T$ decreases and $M$ updates gradually. When the TPS deformation energy reaches minimum, the iterative process terminates and the matching relationship between lumen contours is determined.

\section{Results and discussion}

The methods were tested on in vivo IVUS studies. The image data was acquired using a Jomed Endosonic intravascular imaging device equipped with a $2.9 \mathrm{~F} 30 \mathrm{MHz}$ mechanically driven ultrasonic probe. The imaging catheter was automatically pulled back at a constant speed of $0.5 \mathrm{~mm} / \mathrm{s}$ driven by a motor. A series of tomographic images of the lumen were acquired at a frame rate of $30 \mathrm{fps}$. The image size is $384 \times 384$ pixels, corresponding to $10 \times 10 \mathrm{~mm}$ so that one pixel width is about $26 \mu \mathrm{m}$.

\subsection{Rigid registration}

Figure 4 shows a dissimilarity matrix depicted with a gray-scale image. A bright pixel indicates a large difference between frames. The experimental IVUS study consists of 400 frames so that the size of the image in Fig. 4 is $400 \times 400$ pixels. The dissimilarity matrix presents obvious periodicity. The optimal 
Table 1

Overall accuracy of the rigid registration and offline ECG-gating

\begin{tabular}{ccccccc}
\hline Seq. no. & Number of frames & $\Delta n_{E C G}$ & $\Delta n_{g}$ & $d_{o}$ & $d_{E C G}$ & $d_{g}$ \\
\hline 1 & 1836 & 1 & 1 & 0.2512 & 0.1263 & 0.0831 \\
2 & 1704 & 0 & 1 & 0.2667 & 0.1354 & 0.0769 \\
3 & 2124 & 1 & 2 & 0.2781 & 0.1472 & 0.0721 \\
4 & 1944 & 1 & 1 & 0.2032 & 0.1241 & 0.0693 \\
5 & 2376 & 1 & 3 & 0.2864 & 0.1305 & 0.0874 \\
\hline
\end{tabular}

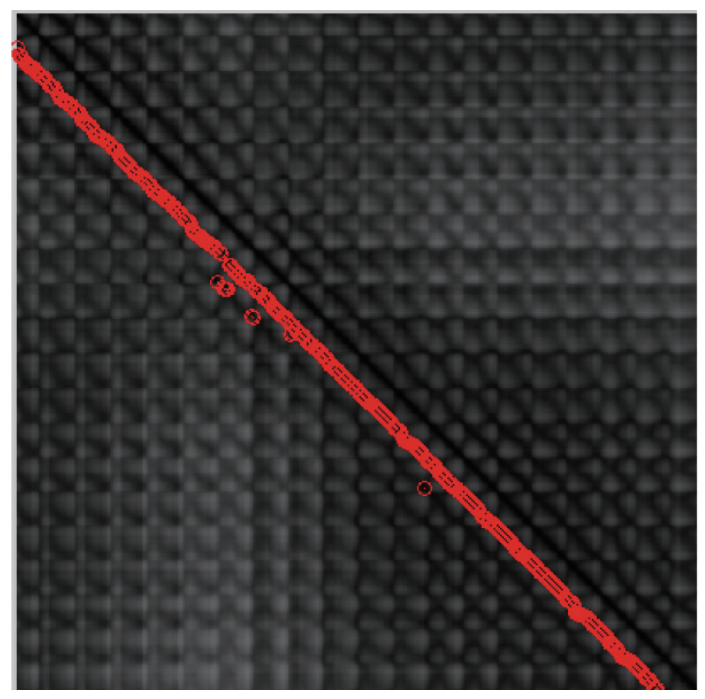

Fig. 4. Optimal paths within the dissimilarity matrix.

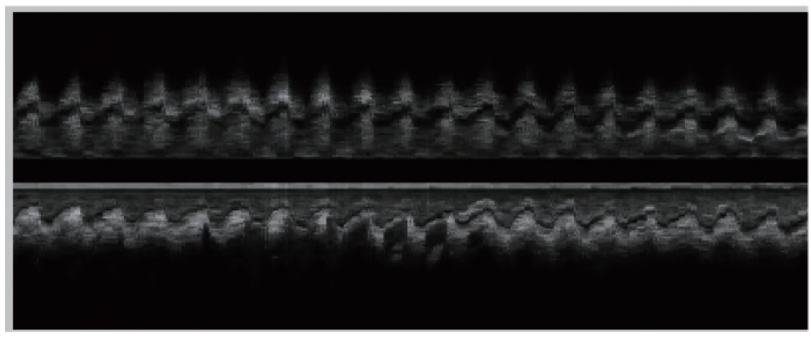

(a)

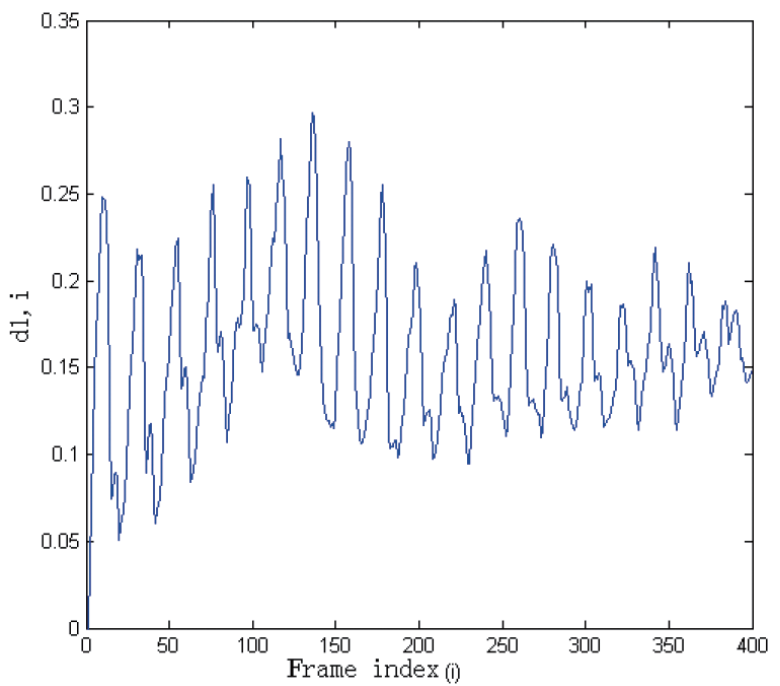

Fig. 5. $d_{1, i} \sim i$ curve.

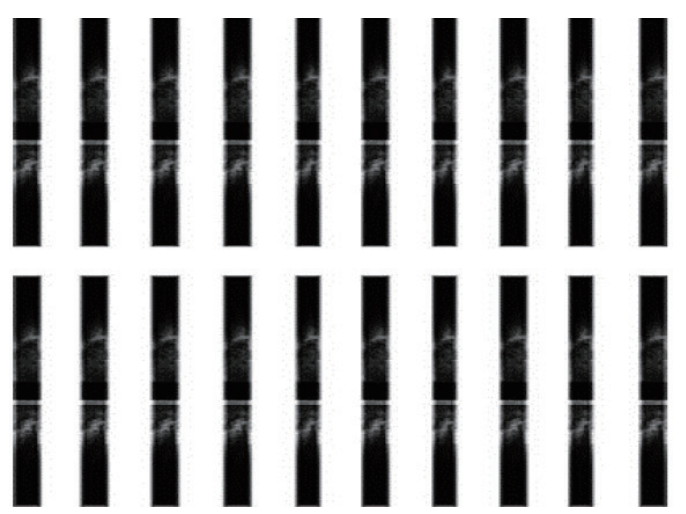

(b)

Fig. 6. (a) Original and (b) registered longitudinal cuts of an IVUS study including 400 frames.

path is represented with a red line. From Fig. 5, one can find that $F=21$. It means that the first frame and $21^{\text {st }}$ frame are matched. Figure 6 shows the sorting results.

We compared the performance of the rigid registration with the off-line ECG-gating. According to synchronously recorded ECG signals, an experienced clinician manually selects those frames collected at the R-wave. Figure 7 indicates that the gated longitudinal cut obtained with the rigid registration 
Table 2

Mean and standard deviation of the elastic registration error measured on five in vivo IVUS frame pairs

\begin{tabular}{ccc}
\hline Frame pair no. & TPS $($ mean \pm SD) & ICP $($ mean \pm SD $)$ \\
\hline 1 & $0.358 \pm 0.952$ & $0.831 \pm 1.894$ \\
2 & $0.568 \pm 1.789$ & $0.747 \pm 1.968$ \\
3 & $0.526 \pm 1.803$ & $0.763 \pm 2.041$ \\
4 & $0.532 \pm 1.671$ & $0.803 \pm 2.251$ \\
5 & $0.519 \pm 1.464$ & $0.778 \pm 2.327$ \\
\hline
\end{tabular}

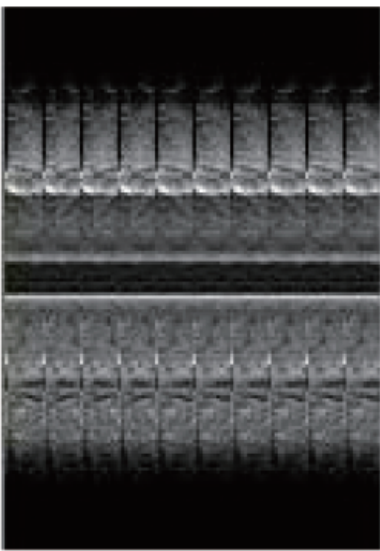

(a)

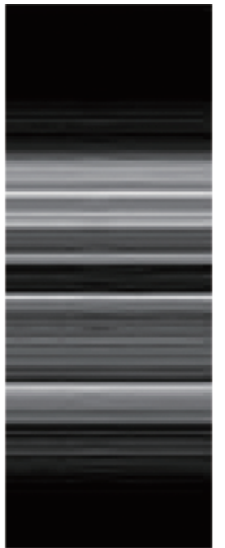

(b)

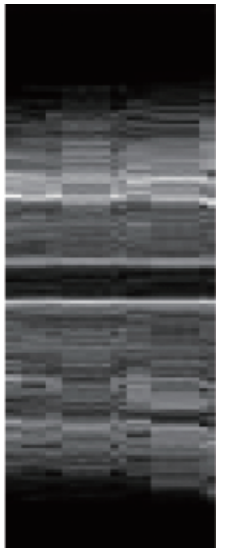

(c)

Fig. 7. Gating result of an IVUS longitudinal cut. (a) Original one; (b) Rigid-registration gated one; (c) ECG-gated one.

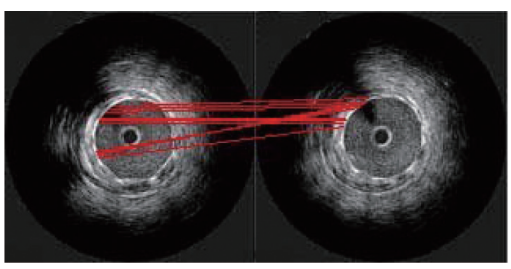

(a)

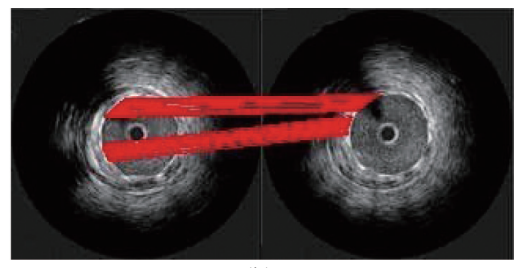

(b)

Fig. 8. Registration results of two consecutive IVUS frames with (a) ICP algorithm and (b) the presented method.

is smoother than the ECG gated one. The average inter-frame dissimilarity and gated frame number difference were used as quantitative scores [5,7] to quantitative evaluate the method. Small scores mean good performance. The gated frame number difference is defined as the absolute difference between the theoretical number of gating frames and the number of selected gating frames. Table 1 lists results of five IVUS studies. In the table, $\Delta n_{E C G}$ and $\Delta n_{g}$ are the gated frame number difference of the ECG gated and rigid-registration gated sequence, respectively. $d_{o}, d_{E C G}$ and $d_{g}$ are the average inter-frame dissimilarity of the original, ECG gated and rigid-registration gated sequence. Obviously, the average inter-frame dissimilarities of the gated sequences are smaller than original ones. Also, the gated frame numbers obtained with rigid-registration and ECG-gating are similar. The average inter-frame difference of rigid-registration gated sequence is smaller than ECG gated ones. 


\subsection{Elastic registration}

Figure 8 provides an example of elastic registration between the calcified plaques in two consecutive frames with the presented method and the popular iterated closest point (ICP) algorithm [12]. Simulated experiments were performed to quantitatively test the accuracy of the registration algorithm. First, an IVUS image was transformed at known displacements. Then, the original image was registered to the pre-transformed image and the displacement error was calculated to evaluate the effectiveness of the registration [13]. Table 2 lists the errors of five pairs of frames denoted with an absolute mean error plus the standard deviation in error less than one pixel width. Clearly, TPS performs better than ICP. For ICP algorithm, the mapping between feature points is determined only based on the nearest distance rule while the one-to-one mapping is ignored. As a result, one-to-many mapping or singularities may appear when large distortion occurs in the image. Furthermore, there are only two types of mapping, 0 and 1, in ICP algorithm. Instead, the mapping coefficient is set as a real number ranging from 0 to 1 in the proposed method to obtain continuous and smooth matching. During the iterative process of ICP, the elastic transformation of feature points is ignored. Instead, the mapping is separated into rigid and non-rigid one in the proposed method. A temperature coefficient, $T$, is set to control the mapping. At the beginning of the registration process, $T$ is large and the rigid mapping accounts for a substantial part. Then, $T$ decrease gradually and the ration of the elastic mapping increases. The registration steps can also be controlled by changing the variation rate of $T$ so that the accurate registration is achieved by balancing the iteration times and steps.

\section{Conclusion}

Methods for rigid and elastic registration for IVUS image sequences have been proposed. Insights from applying the rigid registration methodology suggest that the rigid registration performs better than offline ECG gating. Possible preprocessing procedures including image segmentation or feature extraction are not required. The robustness is ensured by including the variation in the heart rate.

From the proposed elastic registration method, it has been shown that the mapping is smooth and continuous since the mapping coefficient between feature points ranges from 0 to 1 . The elasticity of the registration is ensured by setting a temperature parameter. And the registration steps can also be controlled by adjusting the variation rate of the temperature parameter.

\section{Acknowledgments}

This work was supported by the following funds: 1. National Natural Science Foundation of China grant: 61372042; 2. Fundamental Research Funds for the Central Universities: $2014 Z D 31$.

\section{References}

[1] Leung KYE, Baldewsing R, Mastik F, Schaar JA, Gisolf A, van der Steen AFW. Motion compensation for intravascular ultrasound palpography. IEEE Trans Ultrason Ferroelectr Freq Control. 2006; 53(7): 1269.

[2] Hernàndez-Sabaté A, Gil D, Fernandez-Nofrerías E, Radeva P, Marti E. Approaching artery rigid dynamics in IVUS. IEEE Trans Med Imaging. 2009; 28(11): 1670. 
[3] Bruining N, von Birgelen C, de Feyter PJ, Ligthart J, Li W, Serruys PW, Roelandt JR. ECG-gated versus nongated three dimensional intracoronary ultrasound analysis: implications for volumetric measurements. Cathet Cardiovasc Diagn. 1998; 43(3): 254.

[4] Gatta C, Pujol O, Leor OR, Ferre JM, Radeva P. Robust image based IVUS pullbacks gating. Med Image Comput Comput Assist Interv. 2008; 11(Pt 2): 518.

[5] Rosales M, Radeva P, Rodriguez-Leor O, Gil D. Modeling of image-catheter motion for 3-D IVUS. Med Image Anal. 2009; 13(1): 91

[6] Sun Z and Wang J. Compensation of in-plane rigid motion for in vivo intracoronary ultrasound image sequence. Comput Biol Med. 2013; 43(9): 1077.

[7] O'Malley SM, Granada JF, Carlier S, Naghavi M, Kakadiaris IA. Image-based gating of intravascular ultrasound pullback sequences. IEEE Trans Inf Technol Biomed. 2008; 12(3): 299.

[8] Sun Z and Yan Q. An off-line gating method for suppressing motion artifacts in ICUS sequence. Comput Biol Med. 2010; 40(11-12): 860 .

[9] Sniedovich M. Dynamic programming: foundations and principles. Taylor and Francis Press, 2010.

[10] Katouzian A, Angelini ED, Carlier SG, Suri JS, Navab N, Laine AF. A state-of-the-art review on segmentation algorithms in intravascular ultrasound (IVUS) images. IEEE Trans Inf Technol Biomed. 2012; 16(5): 823.

[11] Rohr K, Stiehl HS, Sprengel R, Buzug TM, Weese J, Kuhn MH. Landmark-based elastic registration using approximating thin-plate splines. IEEE Trans Med Imaging. 2000; 20(6): 526.

[12] Chui H, Rangarajan A. A new point matching algorithm for non-rigid registration. Comput Vis Image Underst. 2003; 89(2-3): 114.

[13] Oakeson KD, Zhu H, Friedman MH. Quantification of cross-sectional artery wall motion with IVUS image registration. In: Proceedings of SPIE International Conference on Medical Imaging 2004: Ultrasonic Imaging and Signal Processing, edited by William F. Walker, Stanislav Y. Emelianov; 2004, p. 119. 